\title{
Universiteit
}

Leiden

The Netherlands

\section{Altered gene expression and root thebaine production in polyploidized and methyl jasmonate-elicited Papaver bracteatum Lindl}

Tarkesh Esfahani, S.; Karimzadeh, G.; Naghavi, M.R.; Vrieling, K.

\section{Citation}

Tarkesh Esfahani, S., Karimzadeh, G., Naghavi, M. R., \& Vrieling, K. (2021). Altered gene expression and root thebaine production in polyploidized and methyl jasmonate-elicited Papaver bracteatum Lindl. Plant Physiology And Biochemistry, 158, 334-341. doi:10.1016/j.plaphy.2020.11.021

Version: $\quad$ Publisher's Version

License: $\quad$ Licensed under Article 25fa Copyright Act/Law (Amendment Taverne)

Downloaded from: https://hdl.handle.net/1887/3264234

Note: To cite this publication please use the final published version (if applicable). 


\title{
Altered gene expression and root thebaine production in polyploidized and methyl jasmonate-elicited Papaver bracteatum Lindl
}

\author{
Saeed Tarkesh Esfahani ${ }^{\mathrm{a}, 1}$, Ghasem Karimzadeh ${ }^{\mathrm{a}, *}$, Mohammad Reza Naghavi ${ }^{\mathrm{b}}$, Klaas Vrieling ${ }^{\mathrm{c}}$ \\ ${ }^{a}$ Department of Plant Genetics and Breeding, Faculty of Agriculture, Tarbiat Modares University (TMU), Tehran, Iran \\ ${ }^{\mathrm{b}}$ Department of Agronomy and Plant Breeding, College of Agricultural and Natural Resources, University of Tehran, Karaj, Iran \\ ${ }^{\mathrm{c}}$ Department of Plant Ecology and Phytochemistry, Institute of Biology, Leiden University, Leiden, the Netherlands
}

\section{A R T I C L E I N F O}

\section{Keywords:}

Papaver bracteatum Lindl.

Papaveraceae

Benzylisoquinoline alkaloid pathway

Induced tetraploidy

Gene expression

HPLC

\begin{abstract}
A B S T R A C T
Persian poppy (Papaver bracteatum Lindl.) is a perennial medicinal plant belonging to the Papaveraceae family that is endemic to the mountainous areas in Northern Iran. It is known for high amounts of the valuable benzylisoquinoline alkaloid thebaine. The effects of induced polyploidy as well as the effect of methyl Jasmonate (MeJA) elicitation on the root production of thebaine and on the expression of five alkaloid biosynthesis related genes were studied. The in vitro tetraploidy induction caused a significant increased expression of norcoclaurine synthase (NCS) and salutaridinol ( $S A T)$, and a significant decreased expression of berberine bridge enzyme (BBE) in the leaves. In the root tissues, the $B B E, N C S$, and $S A T$ showed an increased expression in tetraploid plants, while codeinone reductase $(C O R)$ showed a decreased expression. A similar alteration pattern was found in mixoploid plants when compared to their diploid counterparts. MeJA at concentrations of 0.1 and $0.5 \mathrm{mM}$ caused a remarkable increase in the thebaine content in the roots of treated plants, where the highest thebaine content was identified in plants elicited with $0.5 \mathrm{mM}$ MeJA. Elicitation treatment caused a substantial increase in the expression of NCS and SAT in the leaves, while it had no major effect on BBE, codeine 3-O-demethylase (CODM) and COR. Expression analysis in the roots showed that MeJA caused a significant increase in the expression of only $B B E$ and NCS, while expression of other studied genes remained unchanged. Our results may be exploited for improved thebaine production and the processing of Persian poppy.
\end{abstract}

\section{Introduction}

Persian poppy (Papaver bracteatum Lindl.) is a wild perennial medicinal plant belonging to the section Oxytona of Papaveraceae family that grows in the Alborz Mountains in the North of Iran at altitudes higher than $1800 \mathrm{~m}$ on the slopes facing the Caspian Sea (Sharghi and Lalezari, 1967). It is mainly known for the high amounts of the valuable benzylisoquinoline alkaloid (BIA) thebaine as the main secondary metabolite in roots and capsules (Nyman and Bruhn, 1979), while some 20 other alkaloids are reported to be present at trace amounts only (Wu and Dobberstein, 1977). Thebaine is a secondary metabolite produced through benzylisoquinoline biosynthesis pathway in plant genus Papaver. Other pharmaceutically important alkaloids produced through this pathway, are morphine, codeine, narcotine, oripavine, papaverine, sanguinarine noscapine, etc. (Koukal et al., 2017). In contrast to opium poppy (Papaver somniferum) where morphine is accumulated as the main alkaloid in the capsules, in $P$. bracteatum thebaine as the dominant alkaloid is synthetized or accumulated in different amounts in all plant organs, but with the highest concentration in roots and capsules (Sharghi and Lalezari, 1967; Nyman and Bruhn, 1979). Thebaine serves as a starting material for industrial production of many pharmaceutical drugs, particularly morphine, codeine, semi-synthetic analgesics (oxycodone, oxymorphone, nalbuphine or etorphine) and opioid overdose medications (Koukal et al., 2017). Because of its non-narcotic and non-addictive nature as well as ease of synthetically conversion to other highly-demanded drugs, an increasing international demand exists for thebaine and high-thebaine plant varieties (Shukla et al., 2015).

Biosynthesis of benzylisoquinoline alkaloids (BIAs) in Persian poppy occurs through a well-identified pathway common to several Papaveraceae species including opium poppy (Hodges et al., 1977; Hagel and

\footnotetext{
* Corresponding author. Department of Plant Genetics and Breeding, Faculty of Agriculture, Tarbiat Modares University (TMU), Tehran, Iran.

E-mail address: karimzadeh_g@modares.ac.ir (G. Karimzadeh).

1 Present address: Department of Arid Land and Desert Management, Faculty of Natural Resources and Desert Studies, Yazd University, P.O. Box: 98195-741, Yazd, Iran.
} 
Facchini, 2010) (Fig. 1). It starts with decarboxylation of the amino acid tyrosine by tyrosine decarboxylase (TYDC) followed by the condensation of dopamine and 4-hydroxyphenylacetaldehyde by norcoclaurine synthase (NCS), leading to the production of (S)-norcoclaurine (Samanani and Facchini, 2002). (S)-norcoclaurine is then converted to (S)-Reticuline after four enzymatic reactions catalyzed sequentially by the norcoclaurine 6-O-methyltransferase (6OMT), coclaurine N-methyltransferase (CNMT), (S)-N-methylcoclaurine-3'-hydroxylase (NMCH or CYP80B3) and 3'-hydroxy-N-methylcoclaurine 4'-O-methyltransferase (4'OMT) (Fig. 1). Subsequently, (S)-Reticuline acts as the central precursor for multiple biosynthetic branches, leading to the formation of the main pharmaceutical alkaloids comprising morphine and sanguinarine (Facchini and Park, 2003).

Thebaine is an intermediate product and also the first morphinane alkaloid produced in the benzylisoquinoline alkaloid biosynthesis pathway. In $P$. bracteatum, it is the most abundant alkaloid accumulating in different plant tissues, whereas in P. somniferum it is further processed and converted to morphine via two different pathways catalyzed by codeine 3-O-demethylase (CODM) or thebaine 6-O-demethylase (T6ODM) enzymes (Hagel and Facchini, 2010). Among the genes analyzed in the current study, three (NCS, BBE, and $S A T$ ) were responsible for the enzymes that are involved in the upstream of the thebaine position in the pathway, while two (CODM and COR) are involved in the downstream of the thebaine responsible for further metabolization of this compound (Fig. 1).

The value of $P$. bracteatum could be further increased if the production of thebaine could be enhanced. Increased production of secondary metabolites through polyploidization (Milo et al., 1987; Dhawan and Lavania, 1996; Majdi et al., 2010; Xu et al., 2014; Tavan et al., 2015) and Methyl Jasmonate applications (Lee-Parsons et al., 2004; Aijaz et al., 2011; Afkar et al., 2013; Gurkok et al., 2015), has been frequently reported.

Successful polyploidization has been reported in various medicinal, crop and ornamental plants aimed to produce plants with improved agronomical, phytochemical or economically important characteristics. Production of larger vegetative and reproductive organs including leaves and flowers (Majdi et al., 2010; Miller et al., 2012), increased capability in producing secondary metabolites and medicinally important compounds (Dhawan and Lavania, 1996; Xu et al., 2014) as well as altered and often increased level of expression of important genes and enzymes (Xu et al., 2014; Adams et al., 2003; Mishra et al., 2010) are among the more frequently reported advantages of artificially induced polyploids in various plant species. In vitro polyploidy induction in Papaveraceae species has been previously studied mainly with the aim of obtaining an increased content of pharmaceutical alkaloids. Some authors (Milo et al., 1987) suggested tetraploidization as the most promising approach for development of thebaine-rich poppy lines in $P$. bracteatum. Likewise, in Papaver somniferum L., tetraploidy was

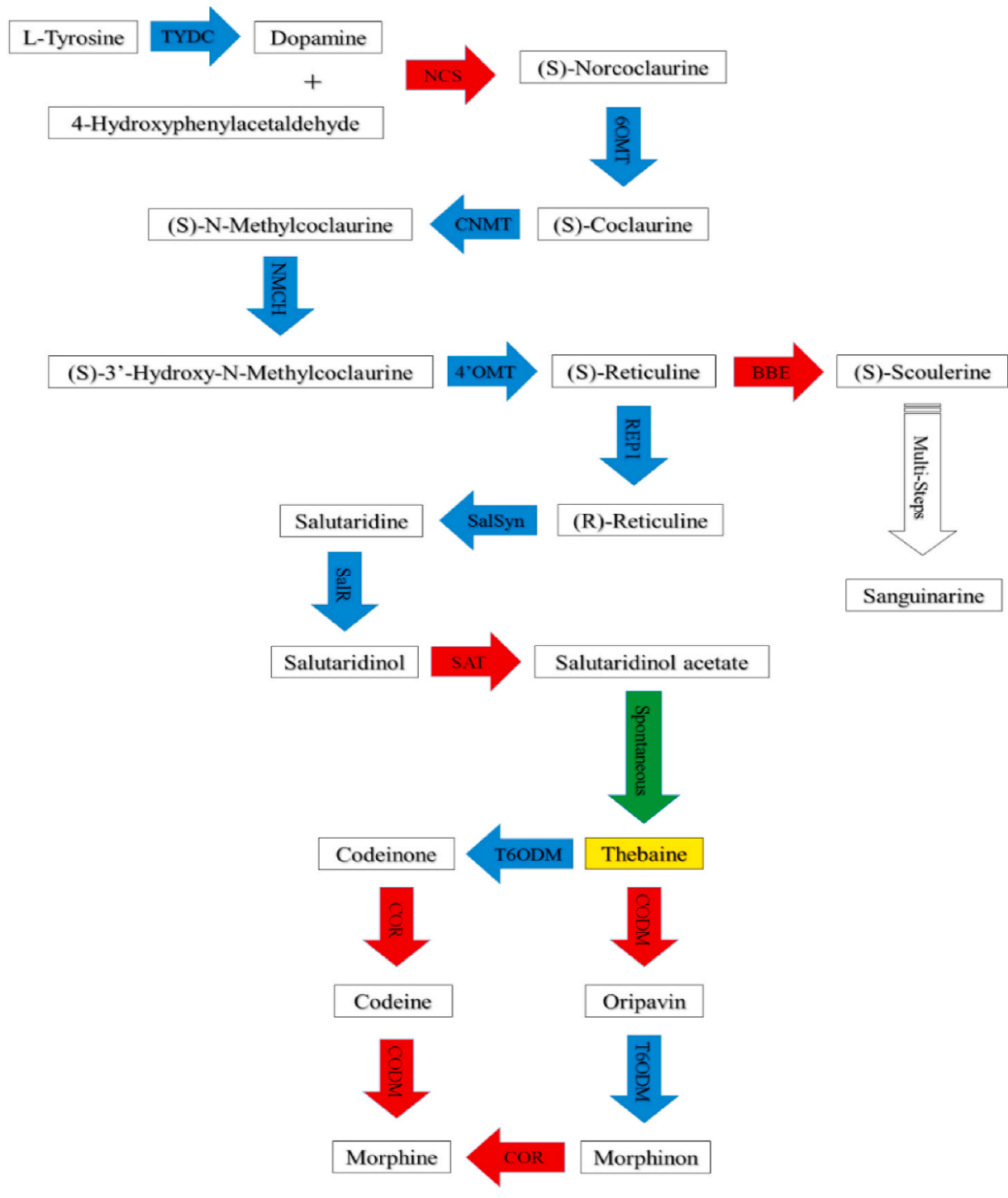

Fig. 1. A brief scheme of the benzylisoquinoline alkaloids pathway. The yellow box indicates the position of thebaine. The blue and green arrows indicate enzymatic and spontaneous reactions respectively. The red arrows show the enzymes of which the genes were selected for expression analysis in this study. Abbreviations include TYDC: tyrosine/DOPA decarboxylase, NCS: norcoclaurine synthase, 6OMT: norcoclaurine 6-O-methyltransferase, CNMT: coclaurine N-methyltransferase, NMCH: N-methylcoclaurine $\quad 3$-hydroxylase, 4'OMT: $\quad 3$ '-hydroxy-N-methylcoclaurine $\quad 4^{\prime}$-Omethyltransferase, BBE: berberine bridge enzyme, REP I: 1,2-dehydroreticuline synthase/reductase, SalSyn: salutaridine synthase, SalR: salutaridine reductase, SAT: salutaridinol 7-O-acetyltransferase, T6ODM: thebaine 6-O-demethylase, CODM: codeine 3-O-demethylase, COR: codeinone reductase. (For interpretation of the references to colour in this figure legend, the reader is referred to the Web version of this article.) 
successfully induced and a significant enhancement in the morphine content and expression level of some important thebaine biosynthesis genes including TYDC, NCS, CNMT, 4OMT, 6OMT, 7OMT, BBE, SAT and COR, was reported (Mishra et al., 2010).

Various biotic and abiotic elicitors have been known as effective means for enhancing the production of different secondary metabolites via in vitro systems, among which methyl jasmonate (MeJA) has been demonstrated as one of the most effective elicitors capable of inducing increased production of various alkaloids (Cho et al., 2007; Holková et al., 2010; Mishra et al., 2013). MeJA has been known to be involved in the signal transduction pathway that induces particular enzymes responsible for catalyzing biochemical reactions which lead to formation of low-molecular weight defense compounds such as polyphenols, alkaloids, quinones, terpenoids, and polypeptides in plants (Zhao et al., 2005). MeJA was also reported as an effective inducer of benzylisoquinoline alkaloid accumulation in plants and cell cultures of $P$. somniferum (Gurkok et al., 2015; Mishra et al., 2013).

Polyploidy induction has been previously reported to cause increased production of thebaine in $P$. bracteatum (Milo et al., 1987). In a previous study, we successfully induced polyploidy in $P$. bracteatum, developed a high-throughput protocol for in vitro tetraploidy induction and analyzed the effects of tetraploidy on various morphological and anatomical traits of the species (Tarkesh Esfahani et al., 2020). In the present study, we aimed to investigate the effects of MeJA elicitation on the production of thebaine in the roots of $P$. bracteatum seedlings. Additionally, in order to understand better the limiting steps in the biosynthetic pathway in induced polyploid as well as MeJA elicited plants, we aimed to measure the gene expression of five important genes in the thebaine biosynthetic pathway. Effects of polyploidy were assessed by comparing tetraploid and mixoploid plants generated in our previous study (Tarkesh Esfahani et al., 2020) against their diploid progenitors. The effect of MeJA was evaluated on newly grown diploid plants of $P$. bracteatum. Specifically, we investigated how the gene expression of the five most important genes involved in the alkaloid biosynthesis pathway changes upon polyploidization and MeJa treatment in $P$. bracteatum, and how the root thebaine production in diploid $P$. bracteatum changes in response to MeJA application.

\section{Materials and methods}

\subsection{Plant material}

Induced autotetraploid and mixoploid plants of $P$. bracteatum as well as their diploid progenitors were provided from plant material developed in our previous work (Tarkesh Esfahani et al., 2020). For gene expression analysis, 15 autotetraploid, 15 mixoploid and their 30 diploid progenitors were selected after their ploidy state was re-confirmed by flow cytometry method. The leaf samples were harvested from the plants in each ploidy class and immediately stored in liquid nitrogen for RNA extraction.

For MeJa elicitation experiment, seeds of mature Persian endemic $P$. bracteatum were collected from Polour region (Latitude $35^{\circ} 52^{\prime} 16.99^{\prime \prime}$ $\mathrm{N}$, Longitude $52^{\circ} 04^{\prime} 38.62$ ' E, Altitude $2489 \pm 50 \mathrm{~m}$ ) on the hillsides of Alborz Mountains in Northern Iran in late July and early August. The seeds of each individual plant were bagged separately. Seeds were planted in plastic seedling trays filled with a combination of $50 \%$ sand, $45 \%$ potting soil and $5 \%$ vermiculite and incubated in growth chamber at $23: 18{ }^{\circ} \mathrm{C}(\mathrm{D}: \mathrm{N})$ and photoperiod of $16: 8 \mathrm{~h}$ (L:D). Plants were watered when needed. After six weeks, 144 plantlets were transferred to $250 \mathrm{ml}$ plastic pots and maintained under the same condition until the MeJA treatment.

\subsection{MeJA treatment}

Plants at the time of treatment were 14 weeks old and had seven to ten developed leaves. The MeJA solution was prepared in four different concentrations $(0,0.1,0.5$ and $2.5 \mathrm{mM})$. The solution with the highest concentration $(2.5 \mathrm{mM})$ was prepared by solving $163.33 \mu \mathrm{lof} 95 \%(\mathrm{w} / \mathrm{v})$ $\left(\mathrm{d}=1.03 \mathrm{~g} \mathrm{ml}^{-1}\right.$ ) Methyl Jasmonate (Sigma Aldrich, Japan) in $300 \mathrm{ml}$ of $0.1 \%$ Triton X-100 (Sigma Aldrich, USA). The 0.5 and $0.1 \mathrm{mM}$ solutions were prepared by diluting the first solution at 1:4 and 1:24 ratios respectively. Then, $200 \mathrm{ml}$ of each solution was sprayed once uniformly on the aerial parts of 36 plants in each concentration treatment group. The control group was sprayed with $200 \mathrm{ml}$ of $0.1 \%$ Triton solution only. After the desired incubation time (24, 48 and 96 h), two symmetrical halves of each leaf and each root sample were taken and weighed; the first half was immediately frozen in liquid nitrogen and kept at $-80{ }^{\circ} \mathrm{C}$ for gene expression analysis, and the second half was freeze dried and kept at $4{ }^{\circ} \mathrm{C}$ until root thebaine measurement analyses.

\subsection{Experimental design and statistical analysis}

The MeJA elicitation experiment was conducted as a factorial experiment based on a completely randomized design. MeJA concentration $(\mathrm{C})$ at four levels $(0,0.1,0.5$ and $2.5 \mathrm{mM})$ and incubation time after treatment $(\mathrm{T})$ at three levels $(24,48$ and $96 \mathrm{~h})$ were considered as the main factors, resulting in 12 treatments in total. Each treatment consisted of four individual plants and was replicated three times. Thus the entire experiment consisted of 144 plants. The effects of MeJA concentration and incubation time after MeJA treatment, on the expression of selected genes as well as the root thebaine content were assessed in this study. Data were analyzed using an analysis of variance, and the mean comparison test based on the least significant difference (LSD, $\alpha=0.05$ ), using SPSS 18 statistical software.

\subsection{HPLC analyses}

Thebaine concentration in the roots of MeJA elicited plants was measured through HPLC analysis. To extract the alkaloids, $1.5 \mathrm{ml}$ of $0.01 \mathrm{M} \mathrm{HCl}$ was added to $100 \mathrm{mg}$ of freeze dried powdered tissue samples and sonicated, using a sonicator bath at room temperature for $30 \mathrm{~min}$ followed by centrifuge at $2500 \mathrm{~g}$ for $10 \mathrm{~min}$. Then, $1000 \mu \mathrm{l}$ of the supernatant was mixed with $400 \mu \mathrm{l}$ of HPLC-grade methanol and loaded on the system after filtration through $0.2 \mu \mathrm{m}$ sterile filter. The thebaine standard with high purity $(\geq 96 \%)$ was supplied by the Agricultural Biotechnology Research Group, University of Tehran. HPLC was carried out using an Agilent 1100 system (Agilent, USA) consisting a C-18 column $(250 \mathrm{~mm} \times 4.6 \mathrm{~mm}, 5 \mu \mathrm{m})$ as the stationary phase. The mobile phase consisted of water:formic acid (1000:1 v/v) as eluent A and acetonitrile:formic acid $(1000: 1, \mathrm{v} / \mathrm{v})$ as eluent $\mathrm{B}$ with a flow rate of 0.8 $\mathrm{ml} \mathrm{min}{ }^{-1}$ and the injection volume of $50 \mu \mathrm{l}$. The gradient condition initiated at 5\% eluent B and increased linearly to $35 \%$ B in $45 \mathrm{~min}$. The chromatograms were acquired at the wavelength of $254 \mathrm{~nm}$ and the thebaine content of the root samples were determined by comparing their retention time and peak area with those of the pure standard.

\subsection{RNA extraction and $q R T-P C R$ analysis}

Based on the phytochemical analysis, the samples belonging to the treatments of 0 (control) and $0.5 \mathrm{mM}$ MeJA, as the two most statistically different treatments, were selected for gene expression analysis aimed at assessing the effect of MeJA elicitation on the expression of genes involved in BIA biosynthesis pathway in $P$. bracteatum. Total RNA was extracted from frozen powdered leaf and root samples, using the method proposed by Sambrook and colleagues (Sambrook et al., 1989) with some modifications. Briefly, 0.5-1 g of grinded material was vortexed for $1 \mathrm{~min}$ in $0.5 \mathrm{ml}$ of extraction buffer $(0.35 \mathrm{M}$ glycine, $0.048 \mathrm{M} \mathrm{NaOH}$, $0.34 \mathrm{M} \mathrm{NaCl}, 0.04 \mathrm{M} \mathrm{EDTA}$, and 4\% SDS) added to $0.5 \mathrm{ml}$ of Tris-equilibrated phenol:chloroform (1:1) solution and centrifuged (12000 g, $5 \mathrm{~min})$, followed by additional vortexing $(1 \mathrm{~min})$ and centrifuging $(12000 \mathrm{~g}, 5 \mathrm{~min})$ the supernatant in Tris-equilibrated phenol $(0.5$ $\mathrm{ml})$ and chloroform $(0.5 \mathrm{ml})$ sequentially. Then, RNA was precipitated 
using one third volume of $\mathrm{LiCl}(8 \mathrm{M})$ overnight at $4{ }^{\circ} \mathrm{C}$ followed by centrifuging $(12000 \mathrm{~g}, 10 \mathrm{~min}$ ), washing in $0.5 \mathrm{ml}$ of $70 \% \mathrm{EtOH}(12000 \mathrm{~g}$, $5 \mathrm{~min}$ ), air-drying and re-suspending the pellet in $20 \mu \mathrm{l}$ water. The isolated RNA was stored at -80 before use.

RNA was quantified, using a NanoDrop spectrophotometer and its quality was evaluated by running on $1.5 \%$ agarose gel, followed by ethidium bromide staining. cDNA was synthesized using iScript ${ }^{\mathrm{TM}}$ Reverse Transcription Supermix kit purchased from Biorad, and stored at $-20^{\circ} \mathrm{C}$.

From the isoquinoline alkaloid pathway, four genes including NCS, $S A T, C O D M$, and COR were selected based on their position in the pathway scheme and their role in production or metabolization of thebaine (Fig. 1). Furthermore, BBE was selected as the first enzyme contributing in the sanguinarine biosynthesis branch.

Gene-specific primers for qPCR analysis were designed using the OligoArchitect online software (Table 2). For CODM no sequence information was available in GenBank and therefore primers for regular PCR were designed based on the sequence already identified in the relative species of $P$. somniferum. Considering the long length of the CODM sequence known in $P$. somniferum (1452 bp), two primer pairs were designed with overlapping segments in order to cover the full length (Table 1).

The PCR products were sequenced and then used for designing qPCR primers (Table 2). The qRT-PCR reactions were carried out using SsoAdvancedTM Universal SYBR $®$ Green Supermix, purchased form Bio-Rad, on a Bio-Rad CFX96 Touch $^{\mathrm{TM}}$ Real-Time PCR detection system. The 18S rRNA served as a reference gene. The mean values of three independent technical and two biological replicates per treatment, were used for normalization of expression data and the fold changes were calculated, using the comparative $\mathrm{Ct}(\Delta \Delta \mathrm{Ct})$ method (Schmittgen and Livak, 2008). The mean comparison analyses for detection of statistically significant differences in gene expression, were conducted on normally distributed $\Delta \mathrm{Ct}$ values from each treatment. The confidence intervals (at 95\% level) of the fold change values were computed as a measure of precision, using Excel (2016) software.

\section{Results and discussion}

\subsection{Gene expression in tetraploid P. bracteatum genes involved in alkaloid biosynthesis}

Compared to diploids, gene expression levels of NCS and SAT increased 6.60 fold and 13.49 fold in tetraploids respectively, while gene expression of BBE was 2 fold decreased in the leaves of $P$. bracteatum. The gene expression levels of CODM and COR in tetraploids were not remarkably changed compared to diploids (Fig. 2a). These results show that the artificial tetraploids alter the expression level of the genes which act the upstream of thebaine in alkaloid biosynthesis pathway (NCS and $S A T)$ while gene expression towards sanguinarine branch $(B B E)$ was unchanged.

Gene expression analysis in the roots of tetraploid P. bracteatum plants showed again that the expression level of genes upstream in the alkaloid pathway (NCS, and SAT) increased in tetraploid plants. In contrast to gene expression of BBE in the shoot, the pathway leading to sanguinarine, is upregulated in the roots. In the roots both genes COR and CODM, downstream of thebaine in the alkaloid pathway, are downregulated in tetraploids (Fig. 2b). Collectively these data suggest that gene expression of genes upstream of thebaine are upregulated while the genes downstream of thebaine are partly downregulated in tetraploids.

It was reported in the previous studies (Milo et al., 1987) that polyploidy induction may cause a significant increase in thebaine production in $P$. bracteatum and that thebaine content in seed capsules of artificial tetraploid plants $(4.9 \%)$ was doubled compared to that in diploid plants $(2.4 \%)$. Nevertheless, overall a slight decrease $(12.42 \%)$ in thebaine yield in capsules of tetraploid plants was identified as a result of the lower number of capsules in tetraploids as compared to diploid progenitors. Here we show that an increased thebaine content in artificial tetraploid plants might be associated with the increased expression of SAT and NCS. These two important genes are therefore potential candidates for further studies in metabolite engineering and breeding for increased thebaine production in P. bracteatum. Cline and Coscia (1988) reported that fungal elicitors produced from Dendryphion penicillatum and Verticillium dahlia pathogens, caused an increased sanguinarine production in diploid $P$. bracteatum cell culture media, however no significant effect was detected on thebaine content. In $P$. somniferum, fungal elicitors including Botrytis cinerea (Holková et al., 2010; Alcantara et al., 2005), Chaetomium globosum, Aspergillus niveoglaucus, Paecilomyces lilacinus, and Trichoderma harzianum (Verma et al., 2014) as well as abiotic elicitors such as methyl jasmonate (Holková et al., 2010), salicylic acid, hydrogen peroxidase and carbon dioxide (Verma et al., 2014), increased sanguinarine content, while causing either a decrease or no significant change in morphine content. Sanguinarine is the final product of the Berberine branch in alkaloid biosynthesis pathway (Fig. 1). The reaction catalyzed by berberine bridge enzyme (BBE) is the first step in this branch, ultimately resulting to sanguinarine. Therefore, it appears that fungal and abiotic elicitors applied on the alkaloid producing plants mainly induce the Berberine branch rather than the branch leading to benzylisoquinoline alkaloids with thebaine and morphine. This suggests that the increase in expression of $B B E$ might be related to the increased sanguinarine production in plants subjected to elicitation.

\subsection{Effect of mixoploidy on the expression level of genes involved in alkaloid biosynthesis}

Gene expression analysis in mixoploid plants can serve as a valuable information for identifying genes which play a more important role in responding to stimuli and elicitation factors.

As in artificial tetraploid $P$. bracteatum, gene expression in mixoploid plants showed that both SAT and NCS are upregulated compared to diploid plants, whereas no change was observed in expression level of $B B E, C O D M$, and COR in leaves (Fig. 2a, c). A similar pattern was exhibited in the root of the mixoploids, except that the gene expression of CODM and COR are not down regulated compared to diploids (Fig. 2 bd). Generally, the results are in line with those obtained in tetraploid plants.

This indicates that the expression of $B B E$ in root tissues in both artificial tetraploids and mixoploids was increased, while no difference in $B B E$ expression level in aerial parts of the polyploid plants was observed compared to diploids. These results are in agreement with those of previous authors who reported a higher expression of $B B E$ in root than in shoot tissue of diploid $P$. bracteatum (Rezaei et al., 2016) and P. somniferum (Facchini, 2006). Considering the fact that sanguinarine is the final product of the berberine branch in alkaloid biosynthesis pathway, $P$. bracteatum can serve as a potential source of sanguinarine production particularly through in vitro strategies, where the employment of polyploidy induction techniques seems promising for increasing

Table 1

Primer pairs designed based on the known sequence of CODM in Papaver somniferum to amplify CODM in Papaver bracteatum.

\begin{tabular}{llll}
\hline Gene & Segment & Forward primer $\left(5^{\prime}>3^{\prime}\right)$ & Reverse primer $\left(5^{\prime}>3^{\prime}\right)$ \\
\hline CODM & 1 & ACTTATCAAGCTAGGCAATGGTTT & GAAAGGCAGAGGGAGTTCTGG \\
& 2 & TTGAATCGGAGGACCAAAGAC & TCACATCCTCATGTAGTCGAGAAA \\
\hline
\end{tabular}


Table 2

qPCR primers for genes involved in alkaloid biosynthesis pathway and for the housekeeping gene (18SrRNA) in Papaver bracteatum.

\begin{tabular}{|c|c|c|c|}
\hline Gene & Forward primer $\left(5^{\prime}>3^{\prime}\right)$ & Reverse primer $\left(5^{\prime}>3^{\prime}\right)$ & Product length (bp) \\
\hline$B B E$ & CGATCCATGAGTCAACTGATTCA & GAGACCACCACCGCTAAG & 89 \\
\hline COR & CTTGGCTTCACTAGGGCAATCGGT & ACTGGAGGGCTGTTGGCTGTC & 86 \\
\hline NCS & AGGCTTCTCAGGGATGTTCTACTT & GTACCGACGCCGCCATTC & 77 \\
\hline$S A T$ & TCCTTGTTTCGACTCGGCATCT & GTATTGGTGGCACTGGCATCC & 80 \\
\hline CODM & ACCAAGTGTTCAGGAATTGGCTAA & GCACCGATATTAGTCAATGGGCTC & 93 \\
\hline 18SrRNA & GTCGTCTCGTCCCTTCTAC & TTCTTCAAAGTAACAGCACCG & 81 \\
\hline
\end{tabular}

A
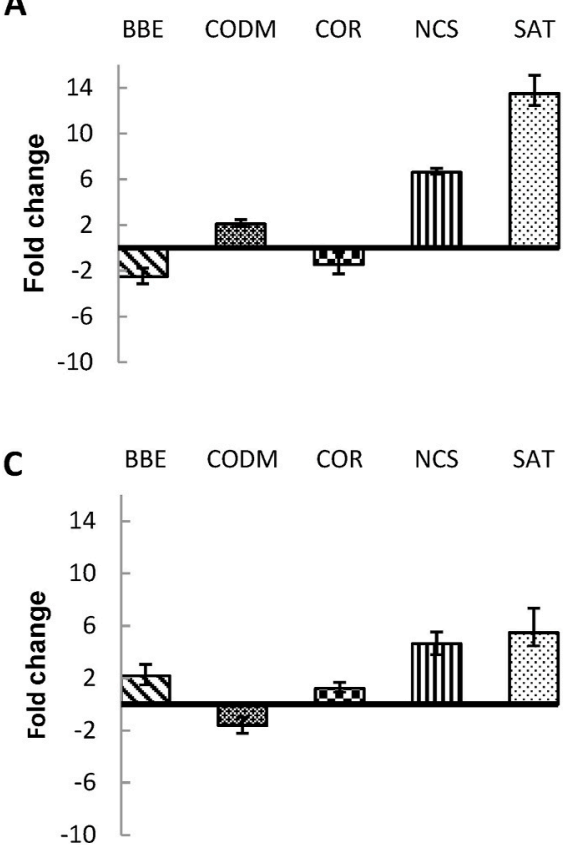

B
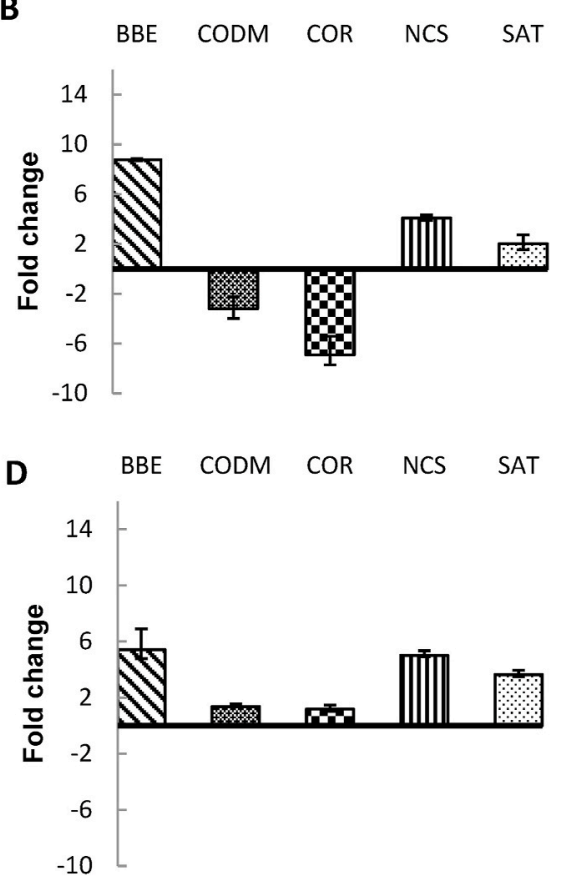

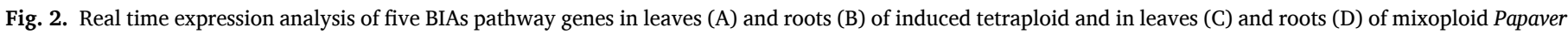

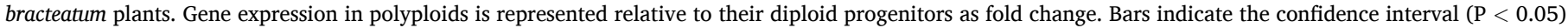
around the mean values.

the yield of this pharmaceutically important alkaloid. Sanguinarine has attracted a great deal of interest for application in medical purposes mainly for its antimicrobial, antifungal, anti-inflammatory and anti-cancer properties (Bertuzzi et al., 2010). Our current results support the potential of $B B E$ gene for further genetic and metabolite engineering studies aimed at achieving a higher amount of Sanguinarine. Furthermore, investigation of the pattern of expression changes in the roots and the leaves of both tetraploid and mixoploid $P$. bracteatum plants indicates the importance of NCS and SAT in thebaine biosynthesis. Additionally, it shows their potential as targets for ploidy breeding and metabolite engineering programs aimed at increasing thebaine yield. Our results are in line with previous authors who reported that the induced increase of the NCS expression through transgenic hairy roots of $P$. bracteatum leads to an increased production of thebaine (Sharafi et al., 2013). Recent studies have showed that although the application of mixoploid plants for large-scale production of secondary metabolites is hard, due to their unstable genetic nature, these plants may perform even better than their tetraploid counterparts in the production of pharmaceutical metabolites under in vitro condition (Tavan et al., 2015). Furthermore, mixoploid plants could be employed as valuable genetic material in ploidy breeding programs particularly for generation of new tetraploid lines through further selection of their pure $4 x$ cells for regeneration and establishment of $4 x$ whole plants (Roy et al., 2001; Chen and Gao, 2007).

\subsection{Effect of MeJA elicitation on the root thebaine content}

In the current study, the effects of two factors including MeJA concentration and incubation time after elicitor treatment on the thebaine content in the roots of diploid $P$. bracteatum plants were examined. Treatment timing and MeJA concentration are two reportedly important factors that have major roles in increasing metabolite accumulation (Lee-Parsons et al., 2004). The concentration of MeJA revealed a significant $(\mathrm{P}<0.05)$ increase on the thebaine content in elicited plants of $P$. bracteatum, while the treatment timing did not cause significant alterations in the thebaine content (Table 3, Fig. 3).

These results support the high importance of MeJA concentration in the induction of thebaine production in plants of $P$. bracteatum. Our results show that MeJA at concentrations of 0.1 and $0.5 \mathrm{mM}$ caused a

Table 3

Results of the analysis of variance for the effect of four MeJA concentrations and three incubation times $(24,46,98 \mathrm{~h})$ after MeJA treatment, as fixed factors, on the root thebaine content in Papaver bracteatum plants.

\begin{tabular}{llll}
\hline Factors & df & MS & F \\
\hline MeJA concentration & 3 & 26.25 & $4.03^{*}$ \\
Incubation time after treatment & 2 & 2.65 & $0.41^{\text {n.s. }}$ \\
Concentration $\times$ incubation time & 6 & 3.80 & $0.58^{\text {n.s. }}$ \\
\hline
\end{tabular}

Note: * and ${ }^{\text {n.s. }}$ indicate significant in $95 \%$ confidence level and insignificance respectively. 


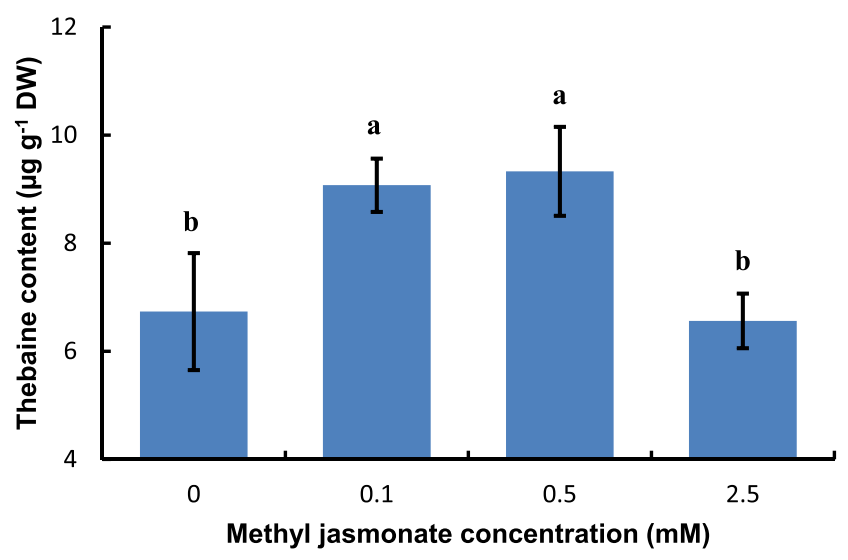

Fig. 3. The effect of methyl jasmonate (MeJA) concentration (mM) on the thebaine content in the roots of Papaver bracteatum plants. Bars indicate the standard error. The columns with the same letter are not significantly different based on the least significant difference (LSD) test at 5\% probability level.

significant increase in the thebaine content in the roots of treated plants (Fig. 3).

Interestingly the highest concentration of MeJa applied ( $2.5 \mathrm{mM})$ did not cause an increased thebaine concentration in the roots, suggesting an optimum curve. The highest thebaine content $\left(9.33 \mu \mathrm{g} \mathrm{g}^{-1} \mathrm{DW}\right)$ was observed in the plants treated by $0.5 \mathrm{mM}$ MeJA. It can also be indicated that the thebaine content showed a gradually increasing trend during the first $48 \mathrm{~h}$ after elicitor application, and then begun to reduce with further progress in time after treatment (Fig. 4).

Considering the results obtained in phytochemical analysis, we selected the samples belonging to the concentration treatments of 0 (control) and $0.5 \mathrm{mM} \mathrm{MeJA}$, as the two most statistically different treatments, for further gene expression analysis, with the aim of assessing the effect of MeJA elicitation on the expression of genes involved in BIA biosynthesis pathway in P. bracteatum.

\subsection{Effect of MeJA elicitation on the expression of BIAs pathway genes}

Gene expression analysis on five important genes involved in BIAs biosynthesis pathway showed that MeJA elicitation caused a significant increase in expression of NCS $(\mathrm{P} \leq 5 \%)$ and $S A T(\mathrm{P} \leq 5 \%)$ in the leaves of elicited $P$. bracteatum plants, while it had no significant effect on the expression level of $B B E, C O D M$, and COR (Fig. 5). On the other hand, the expression analysis in the roots of elicited plants showed that elicitation caused an alteration of only BBE and NCS, while the expression of $S A T$, $C O D M$, and COR remained unchanged. The three to four-fold upregulation of NCS and SAT were apparently sufficient to double the thebaine content in the roots of the plants treated with $0.5 \mathrm{mM}$ MeJA (Fig. 3) despite the fact that BBE was also upregulated 5 folds.

Previous authors have reported that various genes involved in the alkaloid biosynthesis pathway exhibit both developmental and inducible regulation, where some of the genes tend to participate in developmentally controlled pathway, while others are more actively involved in inducible defense mechanisms (Facchini, 2001). It is known that morphine and sanguinarine biosynthesis in the $P$. somniferum species occur by two different pathways and that fungal elicitors applied through cell cultures could induce sanguinarine pathway rather than the pathway leading to morphine (Facchini and Park, 1996). Furthermore, it is reported for $P$. bracteatum species that these types of elicitors cause an increased accumulation of sanguinarine in cell cultures, but no significant change was reported in the content of thebaine as the dominant alkaloid of the species (Cline and Coscia, 1988).

\section{Conclusions}

In this study, the gene expression of NCS and SAT in induced polyploids as well as in MeJA-elicited plants of $P$. bracteatum were upregulated, leading to higher thebaine concentrations. NCS and SAT were recognized as two important genes with a significant contribution in regulating the thebaine content of $P$. bracteatum, while polyploidy induction and MeJA elicitation seem not to cause a significant alteration in the expression of genes involved downstream of thebaine. Also, BBE was found to be involved in the sanguinarine production in the root tissues rather than the shoots tissues. These results showed that polyploidization and MeJA application have an impact on berberine branch (sanguinarine) in the plant root tissues. The increased expression of $B B E$ caused by the MeJA suggests that both thebaine and sanguinarine branches in $P$. bracteatum plants are affected. It confirms that the actual mechanism of induction of metabolic pathways and the exact branches which would be affected as well as the severity of their response to the elicitation factor, might be influenced by the elicitor type (biologic, fungal, hormonal, etc.) and the treatment condition (in vitro, in vivo, cell suspension culture, etc.) under which the elicitor is applied. The thebaine content in the roots of Persian poppy plants tended to increase with MeJA elicitation, where the increase in thebaine content was influenced mainly by the concentration of the elicitor. Our results provide new insights into the knowledge about the ploidy dependent changes in gene expression in plants and may be exploited for improved

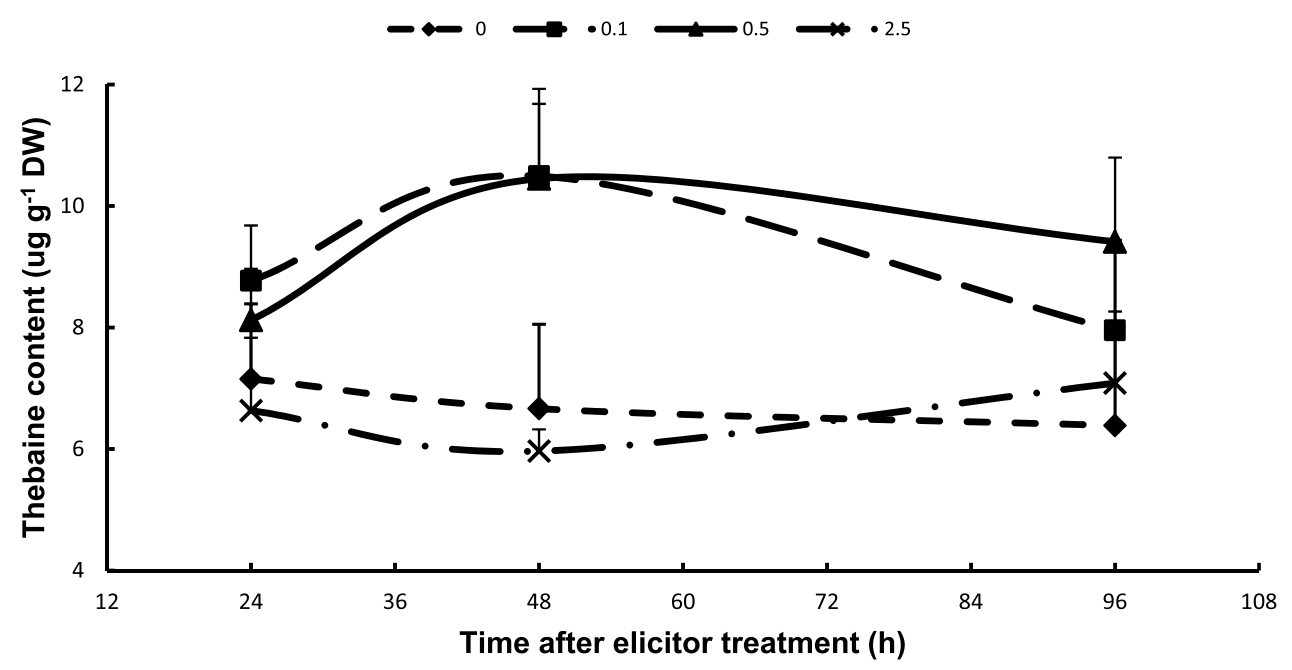

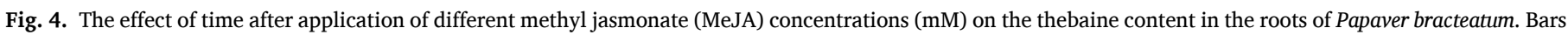
indicate standard errors (ANOVA $\mathrm{F}_{3,24}=4.03, \mathrm{P}<0.05$ ). 

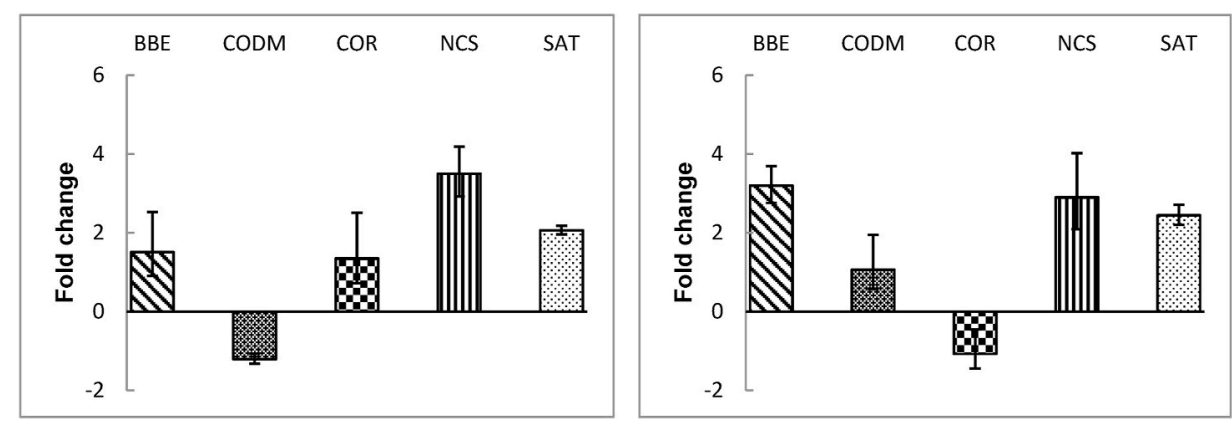

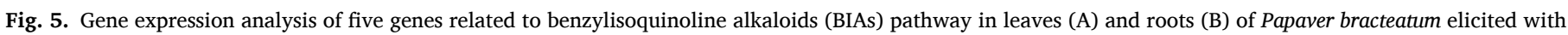

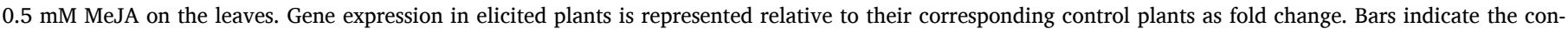
fidence interval $(\mathrm{P}<0.05)$ around the mean values.

production and processing of Persian poppy medicinal plant, and for application in plant breeding and metabolite engineering programs aimed at improving production of thebaine.

\section{Auhtor contributions}

S. Tarkesh Esfahani carried out the experiment, collected data, and wrote the manuscript draft with input from all authors. G. Karimzadeh, M.R. Naghavi and K. Vrieling were involved in planning and supervising the work, design and implementation of the research and final revising the manuscript. All authors contributed to the interpretation of the results, analyzing the data and editing the final manuscript.

\section{Declaration of competing interest}

The authors declare that they have no known competing financial interests or personal relationships that could have appeared to influence the work reported in this paper.

\section{Acknowledgment}

Authors gratefully acknowledge the financial support provided for this survey by the Tarbiat Modares University.

\section{References}

Adams, K.L., Cronn, R., Percifield, R., Wendel, J.F., 2003. Genes duplicated by polyploidy show unequal contributions to the transcriptome and organ-specific reciprocal silencing. Proc. Natl. Acad. Sci. Unit. States Am. 100, 4649-4654.

Afkar, S., Karimzadeh, G., Jalali Javaran, M., Sharifi, M., Behmanesh, M., 2013. Influence of methyl jasmonate on menthol production and gene expression in peppermint (Mentha x piperita L.). J Med Plants By-prod 1, 75-82.

Aijaz, A., Jain, S., Hariharan, A.G., 2011. Effect of elicitation on the production of phytoconstituents through plant tissue culture technique - a review. Int J Drug Disc Herb Res 1, 84-90.

Alcantara, J., Bird, D.A., Franceschi, V.R., Facchini, P.J., 2005. Sanguinarine biosynthesis is associated with the endoplasmic reticulum in cultured opium poppy cells after elicitor treatment. Plant Physiol. 138, 173-183.

Bertuzzi, T., Agosti, B., Gualla, A., Pietri, A., 2010. LC-MS-MS determination of sanguinarine and chelerythrine using a HILIC column. Chromatographia 72, 969-973.

Chen, L.L., Gao, S.L., 2007. In vitro tetraploid induction and generation of tetraploids from mixoploids in Astragalus membranaceus. Sci. Hortic. 112, 339-344.

Cho, H.Y., Lee-Parsons, C.W., Yoon, S.Y.H., Rhee, H.S., Park, J.M., 2007. Enhanced benzophenanthridine alkaloid production and protein expression with combined elicitor in Eschscholtzia californica suspension cultures. Biotechnol. Lett. 29, 2001-2005.

Cline, S.D., Coscia, C.J., 1988. Stimulation of sanguinarine production by combined fungal elicitation and hormonal deprivation in cell suspension cultures of Papaver bracteatum. Plant Physiol. 86, 0161-0165.

Dhawan, O.P., Lavania, U.C., 1996. Enhancing the productivity of secondary metabolites via induced polyploidy: a review. Euphytica 87, 81-89.

Facchini, P.J., 2001. Alkaloid biosynthesis in plants: biochemistry, cell biology, molecular regulation, and metabolic engineering applications. Annu. Rev. Plant Biol. 52, 29-66.

Facchini, P.J., 2006. Regulation of alkaloid biosynthesis in plants. Alkaloids - Chem. Biol. $63,1-44$.
Facchini, P.J., Park, S.U., 1996. The influence of elicitation on the subcellular localization and content of sanguinarine in callus cells of Papaver somniferum L. Biol. Plant. (Prague) 48, 177-192.

Facchini, P.J., Park, S.U., 2003. Developmental and inducible accumulation of gene transcripts involved in alkaloid biosynthesis in opium poppy. Phytochemistry 64, 177-186.

Gurkok, T., Turktas, M., Parmaksiz, I., Unver, T., 2015. Transcriptome profiling of alkaloid biosynthesis in elicitor induced opium poppy. Plant Mol. Biol. Rep. 33, 673-688.

Hagel, J.M., Facchini, P.J., 2010. Dioxygenases catalyze the O-demethylation steps of morphine biosynthesis in opium poppy. Nat. Chem. Biol. 6, 273-275.

Hodges, C.C., Horn, J.S., Rapoport, H., 1977. Morphinan alkaloids in Papaver bracteatum: biosynthesis and fate. Phytochemistry 16, 1939-1942.

Holková, I., Bezáková, L., Bilka, F., Balažová, A., Vanko, M., Blanáriková, V., 2010. Involvement of lipoxygenase in elicitor-stimulated sanguinarine accumulation in Papaver somniferum suspension cultures. Plant Physiol. Biochem. 48, 887-892.

Koukal, P., Hájíček, J., Gupta, S., Hudlický, T., 2017. Model studies toward the total synthesis of thebaine by an intramolecular cycloaddition strategy. Chemistry 2 (26), 7783-7786.

Lee-Parsons, C.W., Ertürk, S., Tengtrakool, J., 2004. Enhancement of ajmalicine production in Catharanthus roseus cell cultures with methyl jasmonate is dependent on timing and dosage of elicitation. Biotechnol. Lett. 26, 1595-1599.

Majdi, M., Karimzadeh, G., Malboobi, M.A., Omidbaigi, R., Mirzaghaderi, G., 2010. Induction of tetraploidy to feverfew (Tanacetum parthenium Schulz-Bip.): morphological, physiological, cytological, and phytochemical changes. Hortscience 45, 16-21.

Miller, M., Zhang, C., Chen, Z.J., 2012. Ploidy and hybridity effects on growth vigor and gene expression in Arabidopsis thaliana hybrids and their parents. G3-Genes Genom Genet. 2, 505-513.

Milo, J., Levy, A., Palevitch, D., Ladizinsky, G., 1987. Thebaine content and yield in induced tetraploid and triploid plants of Papaver bracteatum Lindl. Euphytica 36, 361-367.

Mishra, B.K., Pathak, S., Sharma, A., Trivedi, P.K., Shukla, S., 2010. Modulated gene expression in newly synthesized auto-tetraploid of Papaver somniferum L. South Afr. J. Bot. 76, 447-452.

Mishra, S., Triptahi, V., Singh, S., Phukan, U.J., Gupta, M.M., Shanker, K., Shukla, R.K., 2013. Wound induced tanscriptional regulation of benzylisoquinoline pathway and characterization of wound inducible PsWRKY transcription factor from Papaver somniferum. Plos One 8, e52784.

Nyman, U., Bruhn, J.G., 1979. Papaver bracteatum-a summary of current knowledge. Planta Med. 35, 97-117.

Rezaei, M., Naghavi, M.R., Hoseinzade, A.H., Abbasi, A., 2016. Developmental accumulation of thebaine and some gene transcripts in different organs of Papaver bracteatum. Ind. Crop. Prod. 80, 262-268.

Roy, A., Leggeytt, G., Koutoulis, A., 2001. In vitro tetraploid induction and generation of tetraploids from mixoploids in hop (Humulus lupulus L.). Plant Cell Rep. 20, 489-495.

Samanani, N., Facchini, P.J., 2002. Purification and characterization of norcoclaurine synthase. The first committed enzyme in benzylisoquinoline alkaloid biosynthesis in plants. J. Biol. Chem. 277, 33878-33883.

Sambrook, J., Fritsch, E.F., Maniatis, T., 1989. Molecular Cloning: A Laboratory Manual, second ed. Cold Spring Harbor Press, New York.

Schmittgen, T.D., Livak, K.J., 2008. Analyzing real-time PCR data by the comparative Ct method. Nat. Protoc. 3, 1101-1108.

Sharafi, A., Hashemi Sohi, H., Mousavi, A., Azadi, P., Dehsara, B., Hosseini Khalifani, B., 2013. Enhanced morphinan alkaloid production in hairy root cultures of Papaver bracteatum by over-expression of salutaridinol 7-o-acetyltransferase gene via Agrobacterium rhizogenes mediated transformation. World J. Microbiol. Biotechnol. $29,2125-2131$.

Sharghi, N., Lalezari, I., 1967. Papaver bracteatum Lindl., a highly rich source of thebaine. Nature 213, 1244-1244.

Shukla, S., Mishra, B.K., Mishra, R., Siddiqui, A., Pandey, R., Rastogi, A., 2015. Comparative study for stability and adaptability through different models in developed high thebaine lines of opium poppy (Papaver somniferum L.). Ind. Crop. Prod. 74, 875-886. 
Tarkesh Esfahani, S., Karimzadeh, G., Naghavi, M.R., 2020. In vitro polyploidy induction in Persian Poppy (Papaver bracteatum Lindl.). Caryologia 73, 133-144.

Tavan, M., Mirjalili, M.H., Karimzadeh, G., 2015. In vitro polyploidy induction: changes in morphological, anatomical and phytochemical characteristics of Thymus persicus (Lamiaceae). Plant Cell Tissue Organ Cult. 122, 573-583.

Verma, P., Khan, S.A., Mathur, A.K., Ghosh, S., Shanker, K., Kalra, A., 2014. Improved sanguinarine production via biotic and abiotic elicitations and precursor feeding in cell suspensions of latex-less variety of Papaver somniferum with their gene expression studies and upscaling in bioreactor. Protoplasma 251, 1359-1371.
Wu, F.F., Dobberstein, R.H., 1977. Quantitative determination of thebaine in Papaver bracteatum by high-pressure liquid chromatography. J. Chromatogr. 140, 65-70.

Xu, C.G., Tang, T.X., Chen, R., Liang, C.H., Liu, X.Y., Wu, C.L., Yang, Y.S., Yang, D.P., Wu, H., 2014. A comparative study of bioactive secondary metabolite production in diploid and tetraploid Echinacea purpurea (L.) Moench. Plant Cell Tissue Organ Cult. 116, 323-332.

Zhao, J., Davis, L.C., Verpoorte, R., 2005. Elicitor signal transduction leading to production of plant secondary metabolites. Biotechnol. Adv. 23, 283-333. 